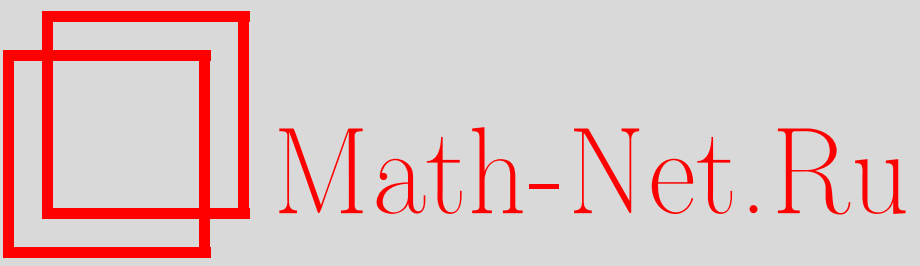

В. А. Зорич, О контактных квазиконформных погружениях, УМH, 2005, том 60, выпуск 2, 161-162

DOI: https://doi.org/10.4213/rm1415

Использование Общероссийского математического портала Math-Net.Ru подразумевает, что вы прочитали и согласны с пользовательским соглашением

http://www.mathnet.ru/rus/agreement

Параметры загрузки:

IP : 54.164 .48 .24

26 апреля 2023 г., $07: 35: 52$ 


\title{
О КОНТАКТНЫХ КВАЗИКОНФОРМНЫХ ПОГРУЖЕНИЯХ
}

\author{
В. А. Зорич
}

1. Введение. Локально обратимое квазиконформное отображение $f: \mathbb{R}^{n} \rightarrow \mathbb{R}^{n}$ при $n>2$ обратимо глобально. Это утверждение было сформулировано М.А. Лаврентьевым [1] для $n=3$ и позднее доказано в работе [2].

Затем оно было развито в нескольких направлениях (см. [3], [4]).

Переход от погружений евклидова пространства к общему случаю погружений римановых многообразий [5], [6] и анализ доказательства в [2] стимулировал появление следующей конформно-инвариантной формы исходной теоремы, связанной с асимптотической геометрией и конформной классификацией римановых многообразий [7].

Для глобальной инбективности квазиконформного погружения $f: M^{n} \rightarrow N^{n}$ риманова многообразия $M^{n}$ в односвязное риманово многообразие $N^{n}$ nри $n>2$ достаточно, чтобы многообразие $M^{n}$ имело конформно-параболический тип.

(О конформном типе многообразия см., например, в [7]-[9].)

В настоящей заметке мы отмечаем, что подобные теоремы имеют место соответственно для квазиконформных погружений группы Гейзенберга и для общих многообразий с контактной структурой конформно-параболического типа.

По-видимому, впервые квазиконформные отображения в контексте контактных структур появились в исследованиях Мостова [10]. Сейчас этому посвящена обширная литература и многочисленные исследования.

За общими геометрическими сведениями, относящимися к неголономной, в том числе к контактной геометрии, мы отсылаем, например, к книге [11] и, в частности, к содержащейся в ней статье Громова.

Основы теории квазиконформных отображений на субримановых многообразиях можно найти в [12]. Экстремалњные длины, емкости конденсаторов и соответствующие оценки можно найти в [13]-[15].

2. Формулировка основных результатов. Мы будем рассматривать пространство $\mathbb{R}^{n}$, $n=2 m+1$, с контактной структурой на нем. Возьмем для определенности евклидово пространство $\mathbb{R}^{3}$ со стандартной контактной структурой, заданной формой $\omega=x d y+d z$.

Нулевые пространства формы образуют распределение $H$ двумерных плоскостей, часто называемых горизонталњными, как и их векторы. Путь в пространстве считается допустимым, или, в иной терминологии, $\omega$-допустимым или горизонтальным, если вектор скорости в любой момент горизонтален.

Заметим, что движение по прямым, параллельным оси $x$, допустимо. В плоскостях $x=c$ допустимы движения вдоль наклонных прямых. Наклон линейно зависит от $c$. Этого достаточно, чтобы стала очевидной возможность соединить любые две точки пространства допустимьм путем.

Итак, пространство точек или состояний $\mathbb{R}^{3}$ с заданным в нем распределением $H$ оказьвается связньм в смысле допустимых переходов из одного состояния в другое.

Если на плоскостях распределения $H$ по тем или иньм причинам имеется риманова метрика (например, как в нашем случае), то пара $\left(\mathbb{R}^{3}, H\right)$ естественно наделяется внутренней метрикой $d$, в которой расстояние между точками измеряется длиной кратчайшего допустимого пути с данными концами. Эта метрика часто назьвается метрикой Карно-Каратеодори ( $C C$-метрикой). Возникает пространство $\left(\mathbb{R}^{3}, H, d\right)$ Карно-Каратеодори (короче, $C C$-пространство $\left.\left(\mathbb{R}^{3}, d\right)\right)$ или кратко $\mathbb{R}_{C C}^{3}$.

В случае связных нечетномерных групп Ли (лево- или право-) инвариантная контактная структура $H$ и многие дальнейшие структуры на $H$ вполне определяются заданием их в касательном пространстве к нейтральному элементу группы (в алгебре Ли).

Классическим примером может служить группа Гейзенберга.

Работа выполнена при поддержке грантов РФФИ-05.01.00981 и НШ-2040.2003.1. 
По отношению к общим многообразиям с контактной структурой (специально по отношению к строго псевдовыпуклым $C R$-многообразиям) она может выполнять примерно ту же функцию локального представления, какую пространство $\mathbb{R}^{n}$ вьполняет в локальном описании $n$-мерного многообразия. Впрочем, в силу теоремы Дарбу все контактные структуры локально изоморфны.

Полученные ниже результаты применимы, в частности, к группе Гейзенберга.

Распределение $H$ может быть связано не обязательно с римановой, а например, с конформной структурой или с $C R$-структурой.

Мы будем рассматривать погружения контактных многообразий, уважающие контактную структуру, т.е. контактные погружения. Точнее, мы будем рассматривать погружения, квазиконформные в смысле $C C$-метрики или конформной структуры. В более общей и простой форме, как показьвают доказательства (см. препринт [16]), мы имеем дело с контактньми отображениями, обладающими некоторым $N$-свойством (нул-свойством), только не по отношению к мере точечных множеств, а по отношению к конформному инварианту - конформному модулю (экстремальной длине) семейств допустимых путей. Класс таких общих погружений обозначим через $N$. Сформулируем, однако, основное утверждение сначала в наиболее конкретном виде.

Теорема 1. Квазиконформное погружение пространства $\mathbb{R}_{C \text { С }}^{3}$ конформно-параболического типа (например, группь Гейзенберга) в себя биективно.

Возможные обобщения, которые, как мы уже отметили, можно увидеть из доказательства, состоят в том, что можно иметь лишь конформную, а не евклидову структуру на $H$; что можно вместо квазиконформности погружения требовать лишь его принадлежность к классу $N$; контактное пространство $\left(\mathbb{R}^{3}, H\right)$ можно заменить любым многообразием $M^{n}$ с контактной структурой $H$ конформно-параболического типа; наконец, можно рассматривать погружение такого многообразия в другое многообразие с контактной структурой.

ТЕОРема 2. Пусть имеются два контактных многообразия одинаковой топологической размерности с конформной структурой на контактных гиперплоскостях, причем первое из многообразий имеет структуру конформно-параболического типа, а второе многообразие односязно. Тогда всякое контактное погружение класса $N$ (в частности, квазиконформное) первого многообразия во второе индективно.

\section{СПИСОК ЛИТЕРАТУРЫ}

[1] М. А. Лаврентьев // Докл. АН СССР. 1938. Т. 20. №4. С. 241-242. [2] В. А. Зорич // Матем. сб. 1967. Т. 74. № 3. C. 417-433. [3] V. A. Zorich // Lecture Notes in Math. 1992. V. 1508. P. 132-148. [4] В. А. Зорич // УМН. 2002. Т. 57. № 3. С. 3-28. [5] M. Gromov // Ann. of Math. Stud. 1981. V. 97. P. 183-213. [6] M. Gromov. Metric Structures for Riemannian and Non-Riemannian Spaces. Boston: Birkhäuser, 1999. [7] В. А. Зорич // Функц. анализ и его прил. 2000. Т. 34. № 3. С. 37-48. [8] В. А. Зорич, В. М. Кесельман // Функц. анализ и его прил. 1996. Т. 30. № 2. С. 40-55. [9] V. A. Zorich // Geom. Funct. Anal. 1999. V. 9. № 2. P. 393-411. [10] G. D. Mostow. Strong Rigidity of Locally Symmetric Spaces. Princeton, NJ: Princeton Univ. Press, 1973. (Ann. of Math. Stud. V. 78.) [11] A. Bellaïche, J. J. Risler (Eds.). Sub-Riemannian Geometry. Basel: Birkhäuser, 1996. (Progr. Math. V. 144.) [12] A. Korányi, H. M. Reimann // Adv. Math. 1995. V.111.№ 1. P. 1-87. [13] A. Korányi, H. M. Reimann // Bull. Sci. Math. (2). 1987. V. 111. №1. P. 3-21. [14] H. M. Reimann // Ann. Acad. Sci. Fenn. Ser. A I Math. 1989. V. 14. № 2. P. 315-324. [15] P. Pansu // Ann. Acad. Sci. Fenn. Ser. A I Math. 1978/79. V. 4. P. 109-118. [16] V. A. Zorich. On contact quasiconformal immersions // Preprint. Zürich: ETH, 2002; www.fim.math.ethz.ch/Preprints.cgi/2002. 